\title{
Pre-hospital ECG for acute coronary syndrome in urban India: A cost-effectiveness analysis
}

Joshua Schulman-Marcus' ${ }^{1}$ Dorairaj Prabhakaran², Thomas A Gaziano $34^{*}$

\begin{abstract}
Background: Patients with acute coronary syndrome (ACS) in India have increased pre-hospital delay and low rates of thrombolytic reperfusion. Use of ECG could reduce pre-hospital delay among patients who first present to a general practitioner (GP). We assessed whether performing ECG on patients with acute chest pain would improve long-term outcomes and be cost-effective.

Methods: We created a Markov model of urban Indian patients presenting to a GP with acute chest pain to compare a GP's performing an ECG versus not performing one. Variables describing the accuracy of a GP's referral decision in chest pain and ACS, ACS treatment patterns, the effectiveness of thrombolytic reperfusion, and costs were derived from Indian data where available and other developed world studies. The model was used to estimate the incremental cost-effectiveness ratio (ICER) of the intervention in 2007 US dollars per quality adjusted life years (QALY) gained.

Results: Under baseline assumptions, the ECG strategy cost an additional \$12.65 per QALY gained compared to no ECG. Sensitivity analyses around the cost of the ECG, cost of thrombolytic, and referral accuracy of the GP yielded ICERs for the ECG strategy ranging between cost-saving and \$1124/QALY. All results indicated the intervention is cost-effective under current World Health Organization recommendations.
\end{abstract}

Conclusions: While direct presentation to the hospital with acute chest pain is preferable, in urban Indian patients presenting first to a GP, an ECG performed by the GP is a cost-effective strategy to reduce disability and mortality. This strategy should be clinically studied and considered until improved emergency transport services are available.

\section{Background}

Ischemic heart disease is already the leading cause of mortality in India [1], and the magnitude of this disease's impact is expected to grow over the next two decades [2]. It is projected that ischemic heart disease will result in two and one-half million Indian deaths by 2020 [3]. Acute coronary syndrome (ACS), including both ST-elevation myocardial infarction (STEMI) and non-ST elevation ACS (NSTE-ACS), is an important manifestation of ischemic heart disease. Rapid diagnosis and treatment with appropriate reperfusion therapies has been proven to increase survival for patients with STEMI. This benefit of reperfusion diminishes as the interval from time of symptom onset to initiation of therapy increases [4]. Current ACS guidelines emphasize the importance of rapid hospital care, especially for

\footnotetext{
* Correspondence: tgaziano@partners.org

${ }^{3}$ Division of Cardiovascular Medicine, Brigham and Women's Hospital,
} Harvard Medical School, USA
STEMI patients who may be eligible for thrombolytic reperfusion within the first twelve hours $[4,5]$.

A recent multi-center Indian registry found only a mean of $58.5 \%$ of Indian STEMI patients received thrombolytics, (6\% of eligible patients undergo percutaneous revascularization) with an average interval between symptom onset and hospital arrival (pre-hospital delay) of five hours [6]. This was twice as long as the median delay seen in the second Euro Heart survey [7]. Increased pre-hospital delay in India has been attributed to poor patient knowledge about ACS, lack of emergency medical services (EMS) infrastructure, and transportation difficulties $[1,6,8,9]$.

In developed countries, pre-hospital electrocardiography (ECG) performed by EMS technicians is associated with faster access to reperfusion therapies for STEMI patients [10]. As urban EMS systems are often lacking in India, ACS patients have been reported as likely to first present to a general practitioner (GP), which has 
generally been associated with increased pre-hospital delay [8,9]. However, one retrospective Indian study of hospitalized ACS patients observed that although overall pre-hospital presentation to a GP doubled the risk of significant pre-hospital delay [8], a subgroup in which the GP performed an electrocardiogram (ECG) had reduced delay compared to patients who did not have an ECG and even to those who presented to the hospital directly. This finding was attributed to improved diagnosis of ACS and more prompt referral of patients to a hospital (unpublished data - with permission from Dr. Rajagopalan 5/25/08). These data were obtained under current urban transportation conditions.

It is therefore plausible that a pre-hospital ECG performed by a GP will have an analogous effect in increasing timely access to reperfusion through quicker and more accurate referral to a hospital. Such a strategy could be useful until improvements are made to India's EMS infrastructure. We modeled the hypothesis that compared to an urban GP not performing an ECG, a GP performing one leads to decreased pre-hospital delay, and consequentially increased eligibility for thrombolytics and improved long-term outcomes. Subsequently, we assessed the cost-effectiveness of this ECG strategy compared to not performing one.

\section{Methods}

\section{Decision-Analytic Model}

We developed a Markov model of urban Indian adult patients presenting to a general practitioner with acute chest pain to assess the overall benefits and costs of the GP performing an ECG versus not performing one (Figure 1). Based on a $2 \%$ incidence rate of chest pain this represents about 8 million patients per year in urban areas in India. The model essentially outlined the survival of patients presenting with chest pain. One influence on survival was receiving appropriate thrombolytics for patients with STEMI. This was improved by both increasing the number of correct referrals from the GP and reducing the delay in presentation to a hospital where thrombolytics could be administered. Another influence on improved mortality was modeled for those with NSTE-ACS who are appropriately admitted to the hospital. Costs were also limited by correct referrals. In the ECG arm of the model, we evaluated the referral decision made by GPs based on clinical history, physical examination, and the ECG. In the other arm, the GP's decision was modeled as based on history and physical examination alone. The cohort was modeled until death, either from cardiovascular disease or from other causes with repeated annual cycles. Updates in risk each year were based on age, ACS history and life-tables for India.

Costs and quality adjusted survival were calculated based on estimates derived from prior studies of a GP's accuracy in referring patients with chest pain to either an appropriate medical facility or home. In both arms, we modeled that STEMI patients incorrectly sent home (false negatives) would be delayed in presenting to a hospital, and consequently would be ineligible for thrombolytic treatment. Patients incorrectly referred to a hospital without ACS (false positives) would incur costs of further medical evaluation. We modeled a cohort of adult patients with the median age of sixty years old, which is the median age of ACS in India [6].

In order to model the clinical effect of the GP performing an ECG, we treated the GP's referral decision as a diagnostic test (Table 1). Sensitivity was the fraction of total patients with ACS who were correctly referred, while specificity was the fraction of total patients without ACS who were correctly sent home by the GP. The baseline sensitivities were derived from published evidence [11-16] with minor adjustments from the Indian observations of Rajagopalan and colleagues (unpublished data).

Specificity of the GP's referral decision was derived from studies performed in developed countries, as no Indian data were available. The specificity of the referral decision when an ECG was administered was estimated from the approximate mean specificity of admission decisions for patients with acute chest pain in the emergency department [12-16]. The specificity of the decision without an ECG was lower owing to decreased diagnostic certainty based on a study of physicians diagnosing acute myocardial infarction without the use of an ECG [17]. We modeled among patients without ACS incorrectly referred to the hospital by the GP (false positives), a fraction equal to one minus the specificity of GP with the ECG would be admitted for 24 hours to "rule-out" ACS.

We adhered to recommendations of the US Panel on Cost-Effectiveness in Health and Medicine in choosing a societal perspective for the baseline analysis [18]. The entire chest pain cohort was modeled until death, either from cardiovascular disease or other causes. No ethics approval was required, because no patients were used for data collection. All analyses were performed with TreeAge Pro Suite 2008 (TreeAge Software Inc., Williamstown, MA). All authors had full access to all the data and had final responsibility for the decision to submit for publication.

\section{Chest Pain and ACS Data}

ACS is the diagnosis in $10-20 \%$ of acute chest pain patients in developed world studies [19-22]. This percentage is unknown in India, so we elected to use the lower bound of $10 \%$ for our baseline analysis. This decision was based on the knowledge that the overall prevalence of coronary disease is likely lower and the 


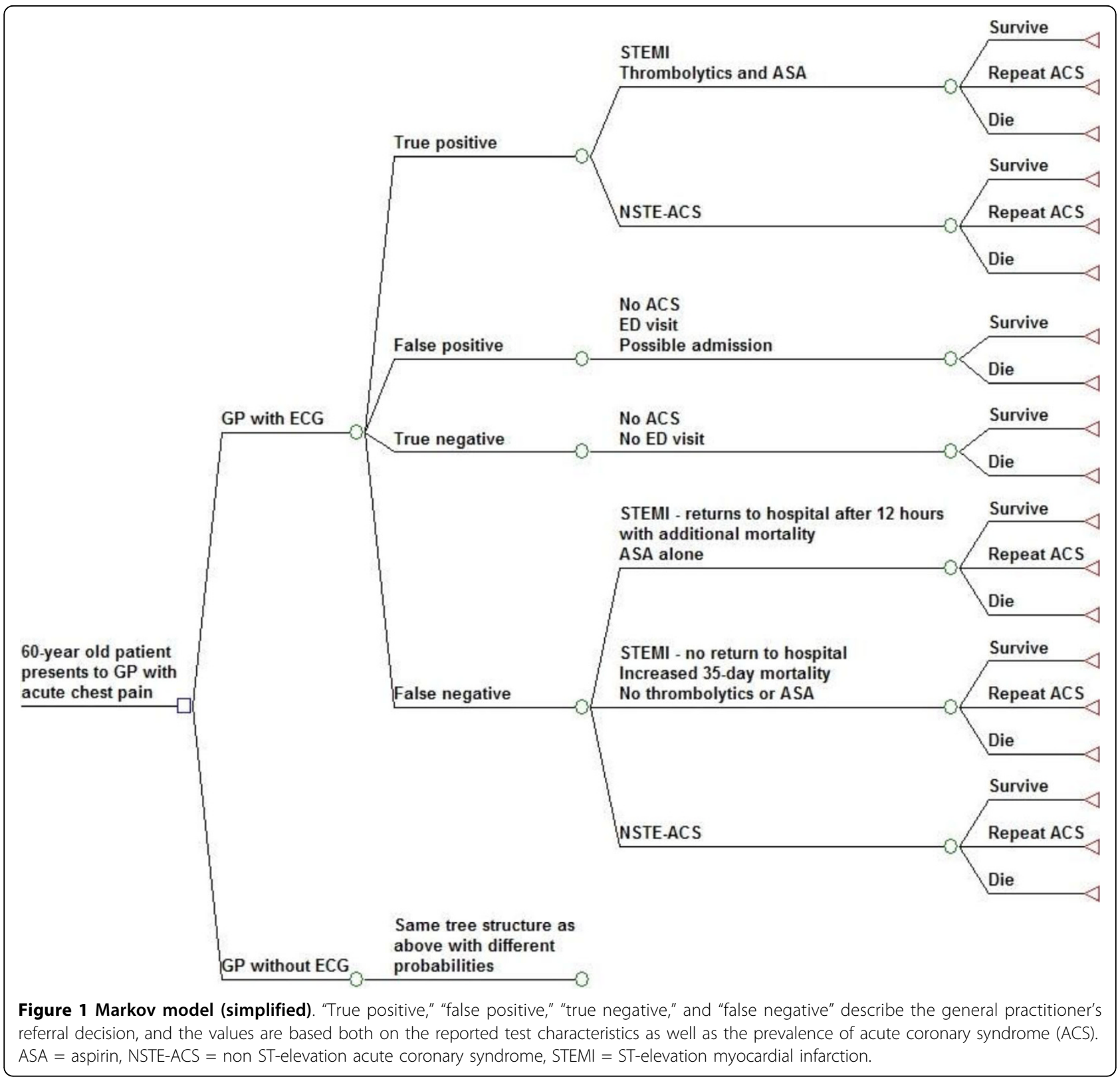

prevalence of other causes of acute chest pain (e.g. tuberculosis) likely greater compared to developed countries. However, we tested the entire range of values in the sensitivity analysis. Based on the recent CREATE registry, which is the largest reported source of ACS data specific to urban India, we modeled $60.6 \%$ as having STEMI and the remainder having NSTE-ACS [6].

The model assumed that $58.5 \%$ of patients with STEMI correctly referred by the GP received thrombolytics, which is the Indian average [6]. As Rajagopalan and colleagues observed a median delay of 24 hours for ACS patients who were misdiagnosed by a GP (unpublished data), we modeled that none of the STEMI patients incorrectly sent home by the GP received thrombolytics regardless of eventual hospitalization status. The shortand long-term effectiveness and risks of thrombolytics were derived from the overall effects reported in the Second International Study of Infarct Survival (ISIS-2) and Fibrinolytic Therapy Trialists (FTT) studies [23-25]. All patients who were treated in a hospital received early aspirin therapy, which had a relative risk reduction derived from ISIS-2.

Mortality to 35 days was estimated from ISIS-2 for STEMI patients [23,24], and from the CREATE registry for NSTE-ACS patients [6]. Patients with STEMI who were incorrectly sent home by the GP but later returned 


\begin{tabular}{lcc}
\hline & Baseline Value & Source(s) \\
\hline Input variables & & 0.1 \\
Chest pain is caused by ACS & 0.818 & Unpublished data from [8,11-16] \\
GP Sensitivity with ECG & 0.667 & Unpublished data from [8] \\
GP Sensitivity without ECG & 0.5 & {$[12-16]$} \\
GP Specificity with ECG & 0.3 & {$[17]$} \\
GP Specificity without ECG & 58.5 & {$[6]$} \\
Correctly referred STEMI patients receiving thrombolysis (\%) & 0.750 & {$[23,24]$} \\
Relative risk reduction of thrombolytics & & {$[37]$} \\
& & {$[39,40,42-44]$} \\
Costs (2007 US\$) & 1.76 & {$[37]$} \\
GP visit & 1.93 & {$[38,45]$} \\
ECG & 3.48 & {$[37]$} \\
Emergency department visit & 117.00 & {$[37]$} \\
Streptokinase & 157.55 & {$[37]$} \\
Admission & 107.67 & {$[37,38]$} \\
Blood transfusion & 211.37 & 16.56 \\
Stroke & & \\
Annual secondary prevention & & \\
\hline
\end{tabular}

ACS = acute coronary syndrome, ECG = electrocardiogram, GP = general practitioner, STEMI = ST-elevation myocardial infarction

Admission costs are for a 5-day admission. The cost of a blood transfusion includes an 3 additional days of hospitalization. The cost of a stroke includes

7 additional days of hospitalization. Annual secondary prevention includes two clinic visits and medications.

to the hospital were modeled to have a twelve-hour prehospital delay conveying an additional $0.9 \%$ mortality, which is half the first-day STEMI mortality rate in the FTT and Clopidogrel and Metoprolol in Myocardial Infarction (COMMIT) studies [25,26]. All ACS patients who were incorrectly sent home by the GP and did not return to the hospital had double the 35-day mortality of those admitted, a hazard determined from developed world studies of ACS patients incorrectly sent home from the emergency department $[11,21]$.

Mortality after 35 days for chest pain patients without ACS was derived from World Health Organization (WHO) life tables for India [27]. We assumed that patients without ACS did not have ischemic heart disease, so we adjusted the annual mortality probabilities to decrease the influence of heart disease for the first ten years. In the patients who had ACS, for the first ten years we used mortality and reinfarction data derived from Law and colleagues [28], with adjustments for the effects of secondary prevention. After ten years, the annual mortality for ACS patients was equivalent to the sum of fatal reinfarction and the yearly mortality in the WHO life tables.

For secondary prevention after 35 days, we considered a regimen of aspirin, a beta-blocker, a statin, and an angiotensin converting enzyme (ACE) inhibitor. As an international survey showed that the actual population of patients taking secondary prevention in India is well below those eligible [29], we modeled the relative risk reduction of secondary prevention only for the percentage of Indian patients taking the individual drugs. The relative risk reductions of the secondary prevention regimen were derived from the reported literature [30-33]. In accordance with evidence that has suggested a diminishing effect of beta-blockers over time [34], the beta-blocker was only included in the secondary prevention regimen for the first six years of the model. We assumed that the relative risk reductions of the drugs were independent, and therefore we calculated the overall effect by multiplying the individual relative risks associated with each drug.

\section{Outcomes and Costs}

Outcomes in the analyses were measured in quality adjusted life-years (QALYs) gained and net health costs. QALYs were obtained by using the disability weights of the WHO Global Burden of Disease Project as used in the 2nd edition of the Disease Control Priorities (DCP) Project [35,36].

Costs of hospital admission, salaries, office visits, and laboratory fees were derived from regional estimates done for the Disease Control Priorities Project [37]. As the median duration of hospitalization for ACS in India is five days [6], we modeled a five day admission to a secondary hospital with the pro-rated labor costs of a cardiologist, junior doctor, nursing care, and an administrative worker. For patients who needed a blood transfusion or stroke care owing to the adverse effects of thrombolytics, additional days of hospitalization were added. Drug costs were calculated from the 
International Drug Price Indicator Guide [38] and local costing data where available. All secondary prevention drug costs were computed as a year's supply, though covering only the proportion of Indian patients taking medical therapy as reported in the WHO-PREMISE survey [29].

The baseline cost of an ECG was estimated as the current reimbursement rate to private hospitals by India's Central Government Health Scheme (CGHS) in large metropolitan areas [39]. An additional $\$ 0.08$ cost was added based on a 500 rupee training cost [40] spread over an estimated 30 annual ECGs performed over a five year period. We did not model the direct costs of transportation to the hospital, time lost from work, or rehabilitation. Initial costs obtained in India rupees prior to 2007 were inflated to 2007 rupees using the Indian wholesale price index [41] and then converted to 2007 US dollars with the midyear exchange rate. The costs are modest but the Gross National Income (GNI) per capita was approximately $\$ 800$ in 2006 , reflecting the relatively cheap labor supply in India and as a result lower costs.

All costs and benefits were tallied at mid-cycle increments. Those dying during a cycle were assumed to incur one half of the utilities and costs of that cycle. All costs as well as health outcomes were discounted at $3 \%$ per year, which is consistent with guidelines [18]. Incremental cost-effectiveness ratios (ICERs) were calculated as the difference in costs between the ECG intervention and no intervention divided by the increase in QALYs gained.

\section{Sensitivity Analysis}

One-way sensitivity analyses on the "sensitivity" and "specificity" of the GP's referral decision relied on studies of admission decisions for emergency department patients with acute chest pain [12-16]. Analyses of the effectiveness of the thrombolytics relied on the standard deviations reported in ISIS-2. As the efficacy of therapy in real Indian settings may be substantially lower than that in developed world controlled trials, we also assessed reduction in thrombolytic efficacy of up to $50 \%$. Sensitivity analyses of the costs of health-care delivery relied on the ranges reported in the DCP working paper [37], except for the cost of an ECG which was estimated from selected Indian price schedules as well as one developed world reimbursement schedule for comparison [42-44]. Analysis of the price of thrombolytic relied on a lower bound from an online Indian database of drug prices [45] and an upper bound from the International Drug Price Indicator Guide. In our sensitivity analyses, we use the term "cost-saving" to mean that the ECG intervention cost less and resulted in more QALYs gained than not performing an ECG. We also performed a probabilistic sensitivity analysis[46] with second-order Monte Carlo simulation of 1000 randomly selected sets of parameters in which we simultaneously sampled values from the distributions with corresponding logit-means and logit-standard deviations.

\section{Results}

Under baseline assumptions, the strategy in which a GP did not perform an ECG resulted in 12.423 QALYs obtained over an average lifetime, while performing an ECG resulted in 12.435 QALYs obtained (Table 2). Approximately $57 \%$ of this gain in QALYs came from the increased use of appropriate thrombolytics, 30\% from increased use of secondary prevention treatment and the remaining $12 \%$ was related to improved hospital survival for those appropriately referred as a result of the ECG. The strategy of not performing an ECG cost $\$ 50.37$ per individual over the lifetime of the cohort, while the ECG intervention cost $\$ 50.52$ per individual. Overall, the ECG intervention had an incremental costeffectiveness ratio (ICER) of $\$ 12.65$ per QALY gained.

One-way sensitivity analyses around the "sensitivity" and "specificity" of the GP's referral decisions resulted in ICERs that ranged between cost-saving to less than \$400/QALY (Table 3). The model was largely insensitive to changes in the referral accuracy (sensitivity) of the GP with an ECG, yielding ICERs from cost-saving to $\$ 103 /$ QALY. When the sensitivity of the GP without an ECG was set to be equal to the baseline value of the GP with the ECG, while holding the specificity constant, the ECG intervention was cost-saving. When the same test was performed for the specificity of the GP without an ECG while holding the sensitivity constant, the ICER was $\$ 351 /$ QALY. If the sensitivity of the GP with ECG was lower than the GP without an ECG then the no ECG strategy could have a ICER of \$580/QALY when compared to the GP with ECG.

Sensitivity analysis around the prevalence of ACS resulted in an ICER that ranged from cost-saving to $\$ 119 /$ QALY (Figure 2). If the ECG's cost was modeled as the maximum Indian cost of approximately $\$ 4$, the ICER of the ECG strategy was approximately $\$ 189$ /

Table 2 Results with baseline assumptions

\begin{tabular}{lccc}
\hline & $\begin{array}{c}\text { Cost } \\
\text { (2007 US } \\
\mathbf{\$})\end{array}$ & $\begin{array}{c}\text { Effects } \\
\text { (QALYs } \\
\text { obtained) }\end{array}$ & $\begin{array}{c}\text { ICER } \\
\text { (\$/QALY } \\
\text { gained) }\end{array}$ \\
\hline $\begin{array}{l}\text { GP without ECG } \\
\text { GP with ECG }\end{array}$ & 50.37 & 12.423 & \\
& 50.52 & 12.435 & \\
$\begin{array}{l}\text { Incremental } \\
\text { Change }\end{array}$ & 0.15 & 0.012 & 12.65 \\
\hline
\end{tabular}

ECG = electrocardiogram, GP = general practitioner, ICER = Incremental costeffectiveness ratio, QALY = quality adjusted life year 


\begin{tabular}{|c|c|c|c|}
\hline & Baseline Value & Tested Range & $\begin{array}{c}\text { ICER }^{*} \\
\text { (\$/QALY gained) }\end{array}$ \\
\hline \multicolumn{4}{|l|}{ Input variables } \\
\hline Chest pain is caused by ACS & 0.1 & $0.01-0.2$ & Cost-savingt - 119 \\
\hline GP sensitivity with ECG & 0.818 & $0.7-0.98$ & Cost-saving - 103 \\
\hline GP sensitivity without ECG & 0.667 & $0.667-0.818$ & 13 - cost-saving \\
\hline GP specificity with ECG & 0.5 & $0.44-0.67$ & 76 - cost-saving \\
\hline GP specificity without ECG & 0.3 & $0.3-0.5$ & $57-351$ \\
\hline Relative risk reduction of thrombolytics & 0.750 & $0.71-0.875$ & $13-12$ \\
\hline \multicolumn{4}{|l|}{ Costs (2007 US\$) } \\
\hline ECG & 1.85 & $1.00-15.00$ & Cost-saving -1124 \\
\hline Streptokinase & 117.00 & $68.74-162.38$ & Cost-saving - 33 \\
\hline Admission & 157.55 & $99.71-459.39$ & Cost-saving - 73 \\
\hline
\end{tabular}

* The ICER is a comparison of the GP with ECG compared to GP without an ECG, with the value on the left corresponding with the leftward most value in the tested range. All ICERs are rounded to the nearest dollar.

† "Cost-saving" here is defined as the ECG intervention costing less and increasing QALYs gained compared to no ECG.

ACS = acute coronary syndrome, ECG = electrocardiogram, ICER = incremental cost-effectiveness ratio, QALY = quality adjusted life year

QALY. When the ECG cost $\$ 15$, about the procedure's cost in the United States [44], the ECG intervention yielded an ICER of $\$ 1124 /$ QALY. The threshold at which the ICER for the cost of the ECG changed from cost-saving to a positive value (cost more and gained more QALYs) was approximately $\$ 2$. Sensitivity analyses on the cost of admission and efficacy of the thrombolytic resulted in minor variation in the baseline ICER.

We performed a probabilistic sensitivity analysis (PSA) on the main variables of uncertainty, including the sensitivity and specificity, the efficacy of thrombolytics in India, the cost of ECGs in India. The PSA using the variables in Table 3 resulted in a mean ICER of $\$ 154 /$ QALY (95\% confidence interval, -\$1790/QALY., \$1830/ QALY). Greater than $97.5 \%$ of the values were less than a willingness to pay threshold of $\$ 3000$.

\section{Discussion and Conclusions}

We modeled a cohort of urban Indian patients with acute chest pain presenting to a general practitioner to compare the lifelong cost-effectiveness of a GP performing an ECG versus not performing one. Our model found that it cost only $\$ 12.65$ to gain an additional quality adjusted life year for this cohort. The relatively cheap

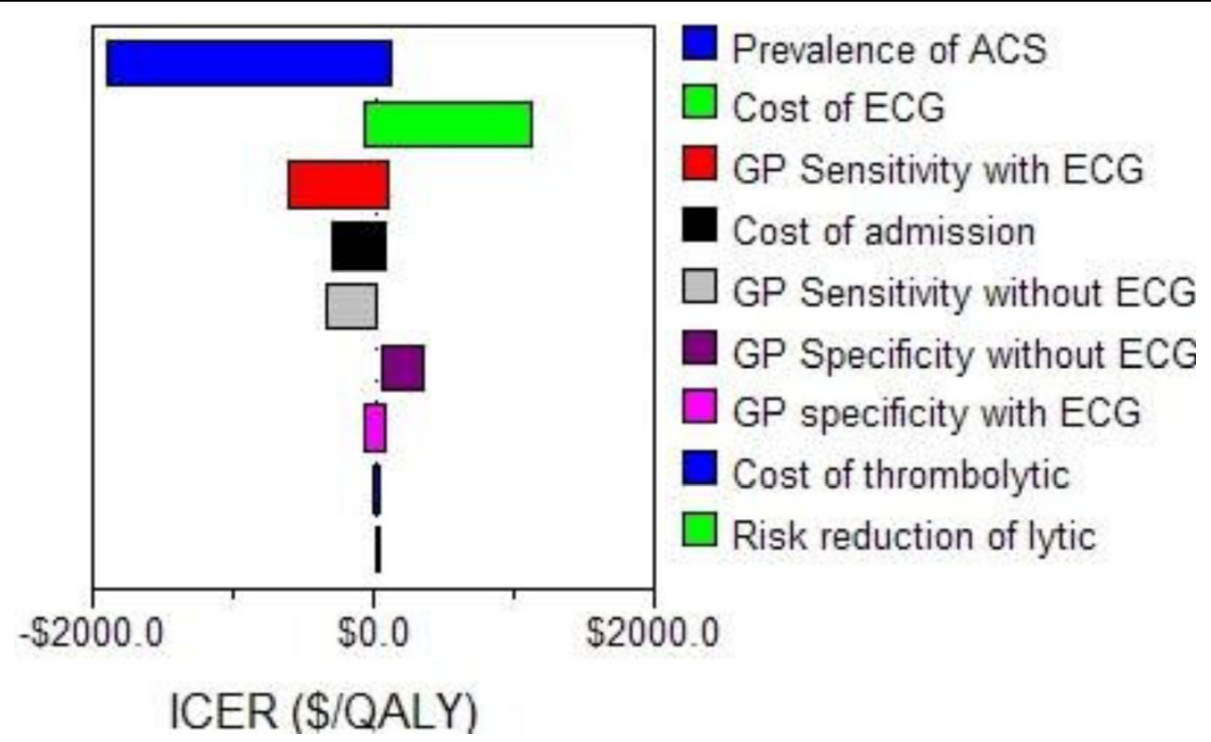

Figure 2 Tornado diagram. This Tornado diagram shows the incremental cost-effectiveness ratio (ICER) of the range of values for each variable tested in the one-way sensitivity analysis. A negative ICER is described in the text as "cost-saving." 
cost for a life year results from the ECG facilitating a GP's decision in the early and appropriate referral of STEMI to a hospital where thrombolysis is administered, as well as a reduction in unnecessary referrals of patients without ACS to hospitals in order to "rule-out" an event. One-way sensitivity analyses around uncertain variables and assumptions demonstrated that this finding is robust, especially for the values most commonly found in contemporary Indian settings.

WHO considers interventions to be cost-effective if they have ICERs that are less than three times gross national income (GNI) per head [47]. In 2006, India's GNI per capita was $\$ 820$, or $\$ 2460$ after adjustment for purchasing power parity [48]. As the baseline findings of our model and all sensitivity analyses suggest the ICER of the GP's performing an ECG compared to not performing one is under $\$ 1200$, the ECG strategy is certainly cost-effective under current WHO definitions. Furthermore, the results of the probabilistic sensitivity analyses suggest the findings are robust with a 95\% confidence interval that is well below the likely willingness to pay threshold for India based on WHO recommendations. Also, since we did not model the direct costs of missed work owing to unnecessary hospitalization of chest pain patients without ACS, it is likely that the ECG strategy is even more cost-effective than our results indicate.

Current guidelines recommend that all patients with acute chest pain should be educated to directly present to a hospital emergency department, and that any such patients presenting to an outpatient practitioner should be urgently referred [4]. We did not model a direct presentation strategy in this study owing to inadequate data, though we think it would be interesting to compare its cost-effectiveness to pre-hospital GP strategies in the future. While such a direct presentation strategy is likely to further increase the population eligible for thrombolysis, it may also cost substantially more owing to increased chest pain patients without ACS presenting to crowded emergency rooms. Still, in the absence of further evidence, we emphasize that direct presentation with acute chest pain is preferable.

In the meantime, while the Indian public undergoes public education regarding the association of chest pain with serious conditions such as myocardial ischemia and the possibility of myocardial infarction and the need to present to an appropriate facility in a timely fashion; the use of ECG machines by general practitioners may facilitate a timely presentation to an appropriate hospital where acute treatments such as thrombolytics can be applied. Training to use the ECG machine and interpret results can be obtained for as little as $\$ 10$ and machines can be purchased for under $\$ 300$. Many private based GPs already may have them. Government based clinics could consider their purchase if not already available. The policy of using ECG machines may not be feasible in rural areas however, given the access to facilities where thrombolysis can be safely be administered may not available.

We did not model a strategy whereby a GP would use a point-of-care troponin assay to make a referral decision. Troponins are frequently negative early in ACS [4] and it is unclear how useful a pre-hospital troponin assay would be when compared to or combined with an ECG. A pre-hospital troponin test's impact on mortality is also uncertain, as troponin assays mostly increase the sensitivity for NSTE-ACS [49], for which thrombolytics are not indicated. We think this is an area that deserves additional research

Sensitivity analyses around the "sensitivity" and "specificity" of the GP's referral decisions with and without the ECG robustly support our baseline findings. We performed these analyses using an especially broad range of possible values for two reasons. First, most data regarding physicians' performance in the diagnosis and referral of ACS patients using ECG are from emergency departments; it is unknown if outpatient GPs perform similarly. Second, there is very limited knowledge about how real physicians perform in the diagnosis and referral of patients with ACS when there is no ECG available. Therefore, in the sensitivity analysis of the GP's referral decision without the ECG, we extended the tested range of values up to the baseline values for the GP with the ECG. This is a very conservative assumption, for it is the equivalent of stating that the ECG is not helpful in referral decision-making, which is at odds with published guidelines [4]. Even so, these one-way analyses did not greatly affect the calculated cost-effectiveness of the ECG strategy

There are several limitations to our study. First, our baseline estimates of the "sensitivity" of a GP's referral decision are based on supplementary observations of a small single-center study that had of mainly urban middle-class patients with confirmed ACS [8]. No confidence intervals were reported in that study. The primary study itself was retrospective, and it was not designed to evaluate a GP's pre-hospital decision making. However, these are the only Indian data available studying a scenario common to urban India. Sensitivity analysis using a wide range of values derived from developed world studies for the sensitivity of GP's referral decision demonstrates that our findings are robust.

Second, owing to limited data on the ultimate diagnosis of acute chest pain across different age groups in India, we only modeled a cohort of sixty-year old patients. We assumed that ACS was the cause of chest pain in $10 \%$ of the cohort, which was the lower end of the reported range in developed world studies [19-22]. However, sensitivity analysis supports our finding that 
the ECG strategy is cost-effective throughout the entire reported range. We found that when ACS as a cause of chest pain was less prevalent, as might be expected in younger cohorts, the ECG strategy was actually cost-saving. When ACS was the cause of acute chest pain in $20 \%$ of the modeled cohort, which may be true in older cohorts of patients, the ECG strategy still had an ICER still well beneath the WHO threshold for cost-effectiveness. Further, a previous study has shown that the use of thrombolytics in the elderly led to an cost-effectiveness ratio for those over the age of 75 what was double of that for those under the age of 70[50] Given that women present at a later age and over all with lower prevalence this would tend to make the ratios in general lower for women than men, especially since case-fatality is higher for females.

Third, owing to limited Indian data, our model did not study the hour-dependent differential effects of thrombolytics on mortality. Major international studies suggest that the subgroup receiving thrombolytics within three hours of ACS onset benefits more than the subgroups receiving them from 3-6 and 6-12 hours after onset $[23,25]$. Our model treated receiving thrombolytics as a binary variable, and the risk reduction in mortality was the average reported from a large international trial. We predict that modeling thrombolytic administration as a time-dynamic variable would lead to even greater reductions in mortality, which would further favor the ECG strategy. This is a topic that deserves further research once Indian data are available.

A recent study in the United States suggests that prehospital ECG performed by emergency medical services increase the frequency and rapidity of reperfusion therapies for patients with STEMI [10]. Given that many Indian ACS patients present first to a GP and the current absence of emergency systems, it is reasonable to study whether a GP performing pre-hospital ECG could yield analogous benefits. We modeled such a GP-based strategy and found it to be robustly cost-effective compared to a GP not performing an ECG for urban patients with acute chest pain. This finding should be considered a strong rationale for clinical trials. Meanwhile, our findings also suggest that it is sensible for GPs in urban India to perform an ECG in patients with acute chest pain until improvements to India's emergency medical services are put in place.

\section{Sources of support}

Sarnoff Cardiovascular Research Foundation (J Schulman-Marcus) Fogarty International Center, National Institutes of Health: K01TW007141-01 (TA Gaziano)

\section{Acknowledgements}

This study was funded through a Sarnoff Cardiovascular Research Foundation fellowship to JSM. The sponsor of the study had no role in study design, data analysis, or the writing of the report. We wish to thank Dr. R Rajagopalan (Chennai) for providing supplementary data.

\section{Author details}

${ }^{1}$ Mount Sinai School of Medicine, One Gustave L Levy Place, New York, NY 10029, USA. ²Department of Cardiology, All-India Institute of Medical Sciences, New Delhi, India. ${ }^{3}$ Division of Cardiovascular Medicine, Brigham and Women's Hospital, Harvard Medical School, USA. ${ }^{4}$ Department of Health Policy and Management, Harvard School of Public Health, Boston, MA 02115 USA.

\section{Authors' contributions}

JSM and TAG - study conception; study design; data collection, analysis, and interpretation; drafting manuscript; decision to publish. DP - data provision; data analysis and interpretation; drafting manuscript; decision to publish. All authors read and approved the final manuscript.

\section{Competing interests}

The authors declare that they have no competing interests.

Received: 21 May 2009 Accepted: 12 March 2010

Published: 12 March 2010

\section{References}

1. Karthikeyan G, Xavier D, Prabhakaran D, Pais P: Perspectives on the management of coronary artery disease in India. Heart 2007, 93(11):1334-1338.

2. Reddy KS: India wakes up to the threat of cardiovascular diseases. J Am Coll Cardiol 2007, 50(14):1370-1372.

3. Ezzati M, Lopez AD, Rodgers A, Murray CJL: Comparative quantification of health risks. Global and regional burden of disease attributable to major risk factors. Geneva: World Health Organization 2004.

4. Antman EM, Anbe DT, Armstrong PW, Bates ER, Green LA, Hand M, Hochman JS, Krumholz HM, Kushner FG, Lamas GA, et al: ACC/AHA guidelines for the management of patients with ST-elevation myocardial infarction: a report of the American College of Cardiology/American Heart Association Task Force on Practice Guidelines (Committee to Revise the 1999 Guidelines for the Management of Patients with Acute Myocardial Infarction). Circulation 2004, 110(9):e82-292.

5. API expert consensus document on management of ischemic heart disease. J Assoc Physicians India 2006, 54:469-480.

6. Xavier D, Pais P, Devereaux PJ, Xie C, Prabhakaran D, Reddy KS, Gupta R, Joshi $P$, Kerkar $P$, Thanikachalam $S$, et al: Treatment and outcomes of acute coronary syndromes in India (CREATE): a prospective analysis of registry data. Lancet 2008, 371(9622):1435-1442.

7. Mandelzweig L, Battler A, Boyko V, Bueno H, Danchin N, Filippatos G, Gitt A, Hasdai D, Hasin Y, Marrugat J, et al: The second Euro Heart Survey on acute coronary syndromes: Characteristics, treatment, and outcome of patients with ACS in Europe and the Mediterranean Basin in 2004. Eur Heart J 2006, 27(19):2285-2293.

8. Rajagopalan RE, Chandrasekaran S, Pai M, Rajaram R, Mahendran S: Prehospital delay in acute myocardial infarction in an urban Indian hospital: a prospective study. Natl Med J India 2001, 14(1):8-12.

9. Malhotra S, Gupta M, Chandra KK, Grover A, Pandhi P: Prehospital delay in patients hospitalized with acute myocardial infarction in the emergency unit of a North Indian tertiary care hospital. Indian Heart J 2003, 55(4):349-353.

10. Diercks DB, Kontos MC, Chen AY, Pollack CV Jr, Wiviott SD, Rumsfeld JS, Magid DJ, Gibler WB, Cannon CP, Peterson ED, et al: Utilization and impact of pre-hospital electrocardiograms for patients with acute ST-segment elevation myocardial infarction: data from the NCDR (National Cardiovascular Data Registry) ACTION (Acute Coronary Treatment and Intervention Outcomes Network) Registry. J Am Coll Cardiol 2009, 53(2):161-166.

11. Lee $T H$, Rouan GW, Weisberg MC, Brand DA, Acampora D, Stasiulewicz C, Walshon J, Terranova G, Gottlieb L, Goldstein-Wayne B, et al: Clinical characteristics and natural history of patients with acute myocardial infarction sent home from the emergency room. Am J Cardiol 1987, 60(4):219-224

12. Pozen MW, D'Agostino RB, Selker HP, Sytkowski PA, Hood WB Jr: A predictive instrument to improve coronary-care-unit admission practices 
in acute ischemic heart disease. A prospective multicenter clinical trial. N Engl J Med 1984, 310(20):1273-1278.

13. Karlson BW, Herlitz J, Wiklund O, Richter A, Hjalmarson A: Early prediction of acute myocardial infarction from clinical history, examination and electrocardiogram in the emergency room. Am J Cardiol 1991, 68(2):171-175.

14. Ting HH, Lee TH, Soukup JR, Cook EF, Tosteson AN, Brand DA, Rouan GW, Goldman L: Impact of physician experience on triage of emergency room patients with acute chest pain at three teaching hospitals. Am J Med 1991, 91(4):401-408.

15. Selker HP, Beshansky JR, Griffith JL, Aufderheide TP, Ballin DS, Bernard SA, Crespo SG, Feldman JA, Fish SS, Gibler WB, et al: Use of the acute cardiac ischemia time-insensitive predictive instrument (ACI-TIPI) to assist with triage of patients with chest pain or other symptoms suggestive of acute cardiac ischemia. A multicenter, controlled clinical trial. Ann Intern Med 1998, 129(11):845-855.

16. Mant J, McManus RJ, Oakes RA, Delaney BC, Barton PM, Deeks JJ, Hammersley L, Davies RC, Davies MK, Hobbs FD: Systematic review and modelling of the investigation of acute and chronic chest pain presenting in primary care. Health Technol Assess 2004, 8(2):iii, 1-158.

17. Herlitz J, Karlson BW, Karlsson T, Dellborg M, Hartford M, Luepker R: Diagnostic accuracy of physicians for identifying patients with acute myocardial infarction without an electrocardiogram. Experiences from the TEAHAT Trial. Cardiology 1995, 86(1):25-27.

18. Gold M, Siegel J, Russell L, M W, eds: Cost-effectiveness in health and medicine. New York: Oxford University Press 1996.

19. Davie AP, Caesar D, Caruana L, Clegg G, Spiller J, Capewell S, Starkey IR, Shaw TR, McMurray JJ: Outcome from a rapid-assessment chest pain clinic. QJM 1998, 91(5):339-343.

20. Burt CW: Summary statistics for acute cardiac ischemia and chest pain visits to United States EDs, 1995-1996. Am J Emerg Med 1999, 17(6):552-559.

21. Pope $J H$, Aufderheide TP, Ruthazer R, Woolard RH, Feldman JA, Beshansky JR, Griffith JL, Selker HP: Missed diagnoses of acute cardiac ischemia in the emergency department. N Engl J Med 2000, 342(16):1163-1170

22. Herlitz J, Karlson BW, Karlsson T, Stensdotter L, Sjolin M: Rate of admission and long-term prognosis among patients with acute chest pain in the 1990s compared with the 1980s. Cardiology 2005, 104(1):51-56.

23. ISIS-2 (Second International Study of Infarct Survival) Collaborative Group: Randomised trial of intravenous streptokinase, oral aspirin, both, or neither among 17,187 cases of suspected acute myocardial infarction: ISIS-2. Lancet 1988, 2(8607):349-360.

24. Baigent C, Collins R, Appleby P, Parish S, Sleight P, Peto R: ISIS-2: 10 year survival among patients with suspected acute myocardial infarction in randomised comparison of intravenous streptokinase, oral aspirin, both, or neither. The ISIS-2 (Second International Study of Infarct Survival) Collaborative Group. BMJ 1998, 316(7141):1337-1343.

25. Fibrinolytic Therapy Trialists' (FTT) Collaborative Group: Indications for fibrinolytic therapy in suspected acute myocardial infarction: collaborative overview of early mortality and major morbidity results from all randomised trials of more than 1000 patients. Lancet 1994, 343(8893):311-322.

26. Chen ZM, Pan HC, Chen YP, Peto R, Collins R, Jiang LX, Xie JX, Liu LS: Early intravenous then oral metoprolol in 45,852 patients with acute myocardial infarction: randomised placebo-controlled trial. Lancet 2005, 366(9497):1622-1632.

27. Rodriguez T, Malvezzi M, Chatenoud L, Bosetti C, Levi F, Negri E, La Vecchia C: Trends in mortality from coronary heart and cerebrovascular diseases in the Americas: 1970-2000. Heart 2006, 92(4):453-460.

28. Law MR, Watt HC, Wald NJ: The underlying risk of death after myocardial infarction in the absence of treatment. Arch Intern Med 2002, 162(21):2405-2410

29. Mendis S, Abegunde D, Yusuf S, Ebrahim S, Shaper G, Ghannem H, Shengelia B: WHO study on Prevention of REcurrences of Myocardial Infarction and StrokE (WHO-PREMISE). Bull World Health Organ 2005, 83(11):820-829.

30. Antithrombotic Trialists' Collaboration: Collaborative meta-analysis of randomised trials of antiplatelet therapy for prevention of death, myocardial infarction, and stroke in high risk patients. BMJ 2002, 324(7329):71-86
31. Dagenais GR, Pogue J, Fox K, Simoons ML, Yusuf S: Angiotensinconverting-enzyme inhibitors in stable vascular disease without left ventricular systolic dysfunction or heart failure: a combined analysis of three trials. Lancet 2006, 368(9535):581-588.

32. Freemantle N, Cleland J, Young P, Mason J, Harrison J: Beta Blockade after myocardial infarction: systematic review and meta regression analysis. BMJ 1999, 318(7200):1730-1737.

33. The Long-Term Intervention with Pravastatin in Ischaemic Disease (LIPID) Study Group: Prevention of cardiovascular events and death with pravastatin in patients with coronary heart disease and a broad range of initial cholesterol levels. N Engl J Med 1998, 339(19):1349-1357.

34. Yusuf S, Peto R, Lewis J, Collins R, Sleight P: Beta blockade during and after myocardial infarction: an overview of the randomized trials. Prog Cardiovasc Dis 1985, 27(5):335-371.

35. Jamison D, Breman J, Measham A, Alleyne G, Claeson M, Evans DB, et al: Disease control priorities in developing countries. New York: Oxford University Press and the World Bank, 22006.

36. Mathers $C D$, Lopez AD, Murray $C J L$ : The burden of disease and mortality by condition: data, methods, and results for 2001. Global Burden of Disease and Risk Factors New York: Oxford University Press 2006, 45-93.

37. Mulligan J, Fox-Rushby J, Taghreed A: Unit costs of health care inputs in low and middle income regions. Disease Control Priorities Project Working Paper 9, Bethesda (MD) 2004.

38. Fyre J, ed: International drug price indicator guide. Cambridge (MA): Management Sciences for Health 2008.

39. Ghaffar A, Reddy KS, Singhi M: Burden of non-communicable diseases in South Asia. BMJ 2004, 328(7443):807-810.

40. Shafey O, Eriksen M, Ross H, Mackay J: The Tobacco Atlas, Third Edition. Atlanta: American Cancer Society 2009.

41. Ahmad N, Bhopal R: Is coronary heart disease rising in India? A systematic review based on ECG defined coronary heart disease. Heart 2005, 91(6):719-725.

42. Goyal A, Yusuf S: The burden of cardiovascular disease in the Indian subcontinent. Indian J Med Res 2006, 124(3):235-244.

43. Jafar TH, Qadri Z, Chaturvedi N: Coronary artery disease epidemic in Pakistan: more electrocardiographic evidence of ischaemia in women than in men. Heart 2008, 94(4):408-413.

44. Joshi R, Chow CK, Raju PK, Raju R, Reddy KS, MacMahon S, Lopez AD, Neal B: Fatal and Nonfatal Cardiovascular Disease and the Use of Therapies for Secondary Prevention in a Rural Region of India. Circulation 2009, 119(14):1950-1955.

45. Yusuf S, Hawken S, Ounpuu S, Dans T, Avezum A, Lanas F, McQueen M, Budaj A, Pais P, Varigos J, et al: Effect of potentially modifiable risk factors associated with myocardial infarction in 52 countries (the INTERHEART study): case-control study. Lancet 2004, 364(9438):937-952

46. Doubilet $P$, Begg CB, Weinstein MC, Braun P, McNeil BJ: Probabilistic sensitivity analysis using Monte Carlo simulation. A practical approach. Med Decis Making 1985, 5(2):157-177.

47. Macroeconomics and health: investing in health for economic development. Geneva: World Health Organization 2001.

48. S Mauss FB, Schmutz G, Henke J, Richter WO: Lipoprotein(a) in patients initiating antiretroviral therapy. HIV Medicine 2008, 9(6):415-420.

49. Roger VL, Killian JM, Weston SA, Jaffe AS, Kors J, Santrach PJ, TunstallPedoe $\mathrm{H}$, Jacobsen SJ: Redefinition of myocardial infarction: prospective evaluation in the community. Circulation 2006, 114(8):790-797.

50. Gaziano TA: Cardiovascular Disease in the Developing World and Its Cost-Effective Management. Circulation 2005, 112(23):3547-3553.

\section{Pre-publication history}

The pre-publication history for this paper can be accessed here:http://www. biomedcentral.com/1471-2261/10/13/prepub

doi:10.1186/1471-2261-10-13

Cite this article as: Schulman-Marcus et al:: Pre-hospital ECG for acute coronary syndrome in urban India: A cost-effectiveness analysis. BMC Cardiovascular Disorders 2010 10:13. 